\title{
Helical Molecular Springs under High Pressure
}

\author{
Jiaxu Liang, ${ }^{a}$ Cheng-Wei Ju, ${ }^{b}$ Wenhao Zheng, ${ }^{a}$ Manfred Wagner, ${ }^{a}$ Zijie Qiu, ${ }^{a, \star}{ }^{*}$ Tanja Weil,,${ }^{a, \star}$ and \\ Klaus Müllen ${ }^{\mathrm{a}, \mathrm{c}, *}$
}

[a] Dr. J. Liang, W. Zheng, Dr. M. Wagner, Dr. Z. Qiu, Prof. Dr. T. Weil, Prof. Dr. K. Müllen

Max Planck Institute for Polymer Research

Ackermannweg 10, 55128 Mainz, Germany

E-mail: muellen@mpip-mainz.mpg.de, weil@mpip-mainz.mpg.de, qiu@mpip-mainz.mpg.de

[b] C.-W.Ju

Pritzker School of Molecular Engineering, University of Chicago

Chicago, Illinois 60637, USA

[c] Prof. Dr. K. Müllen

Department of Chemistry, University of Cologne

Greinstr. 4-6, 50939 Cologne, Germany

Abstract: Although the unique structure of helicenes resembles molecular springs, the effects of pressure on their extensioncontraction cycles have rarely been explored. Herein, we investigated the fluorescence of two $\pi$-extended [ $n$ ] helicenes with different helical lengths $n$, here named [7] and [9], under high pressure in a diamond anvil cell. Based on experimental results and theoretical calculations, the mechanical and fluorescent properties of the molecular springs were found to be influenced not only by the intermolecular packing, but also by the intramolecular $\pi-\pi$ interactions between their overlapping helixes. As a more rigid molecular spring, [9] exhibited a more sensitive response of its fluorescence to hydrostatic pressure than [7]. Our results provide new insights into structure-property relationships under high-pressure conditions and verify the potential of helicenes as molecular springs for future applications in molecular machines.

[ $n$ ] Helicenes are non-planar polycyclic aromatic hydrocarbons (PAHs) with $n$ arylene rings fused in their ortho positions ${ }^{[1]}$. The helically twisted structures lead to a series of exciting mechanical, electronic, and optical properties ${ }^{[2]}$. More intriguing, such unique helical structures have prompted scientists to envision applications as molecular springs in molecular machines. Investigating the elastic extension-contraction motion under external stimuli is of great importance for the development of helical smart materials toward sensing, chemical separation, and nano-mechanical actuators ${ }^{[3]}$. Experimental and theoretical studies have, however, remained rare. By step-wise chemical reduction with alkali metals, double helicenes could be successively compressed, exhibiting profound bond length alterations and conformational changes ${ }^{[4]}$. Recently, Hirose and Matsuda synthesized a flexible $\pi$-expanded helicene and described it as a soft molecular spring based on density functional theory (DFT) calculations ${ }^{[5]}$. According to their simulations, the potential energies of different helicenes increase upon elongation, while the force constants of these molecular springs strongly depend on their helical diameters. There is, thus, a striking similarity to the mechanical properties of macroscopic spring materials in daily life. However, experimental verification of such a theory is very challenging due to the difficulties of directly measuring the molecular deformation and potential energy under applied forces. Alternative approaches are urgently needed.

With the development of high-pressure technology ${ }^{[6]}$, many experiments have been conducted to study the structure-property relationships of luminescent materials under pressure, including polycyclic aromatic molecules ${ }^{[7]}$, luminogens with rotationally and/or vibrationally mobile groups ${ }^{[8]}$, donor-acceptor type fluorophores ${ }^{[9]}$, and carbon dots ${ }^{[10]}$. Various mechanisms have been proposed to explain the emission behavior of different compounds upon compression such as increased $\pi-\pi$ stacking interactions ${ }^{[11]}$, formation of excimers or exciplexes ${ }^{[12]}$, enhanced intermolecular hydrogen bonds ${ }^{[13]}$, planarization of twisted molecular conformations ${ }^{[14]}$, or promotion of intramolecular charge transfer effects ${ }^{[9 b]}$. However, high-pressure studies for helically twisted PAHs, which could reflect both inter- and intramolecular $\pi-\pi$ interactions, remain unexplored until now. Measuring the fluorescence of helicenes under high pressure is expected to shed light on structural changes and thus experimentally explore their potential as molecular springs.
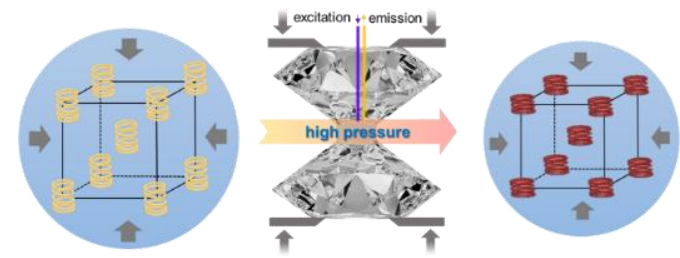

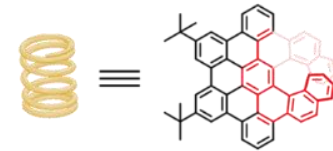

[7] or

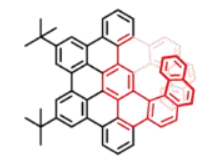

[9]

Figure 1. Illustration of applying high pressure on molecular springs [7] and [9] using a diamond anvil cell. 
Recently, we synthesized a pair of $\pi$-extended [ $n]$ helicenes $(n=7$ and 9$)$ with the same $\pi$-extension motif, but different helical lengths $n^{[15]}$. In this work, these m-extended helicenes (denoted as [7] and [9], respectively) were selected as models of helical molecular springs and their fluorescence signals were utilized to investigate the structural changes under high pressure in a diamond anvil cell (DAC) (Figure 1). Based on our experimental results, we found that [9], as a longer and more rigid molecular spring, showed a larger shift in its fluorescence wavelength than [7] in the crystalline state and dilute solution. Furthermore, the unique intramolecular $\pi-\pi$ interaction between overlapping was demonstrated to affect both their mechanical and fluorescent properties. The different structure-property relations of [7] and [9] under pressure were further elucidated by theoretical calculations using density functional theory (DFT) and hybrid quantum mechanics/molecular mechanics (QM/MM) methods.

Adopting the calculation approach of Hirose and Matsuda ${ }^{[5]}$, the spring-like mechanical characteristics of [7] and [9] upon compression were investigated by simulations of their potential energy in vacuum. The distance ( $r$ ) between the two centroids of the overlapping benzene rings at the helixes was optimized to be 3.90 and $3.54 \AA$ for [7] and [9], respectively (Figure S1) in the pristine state ${ }^{[15]}$. The potential energy $(E)$ was calculated to change in an approximately linear correlation to the square of distance change $(\Delta r)^{2}$, which matched with the function of spring energy on the macroscopic level (Figure S2):

$$
E=\frac{1}{2} k(\Delta r)^{2}
$$

Therefore, the force constants $(k)$ of the molecular springs were determined as 29.0 and $88.3 \mathrm{~N} \mathrm{~m}^{-1}$ for [7] and [9], respectively, suggesting that [9] is a much more rigid molecular spring with a larger force constant.

After growing the crystals of [7] and [9] by solvent diffusion ${ }^{[15]}$, the crystalline samples were loaded in the chamber of the DAC apparatus. Since [7] and [9] are emissive in the crystalline state with peaks centred at $545 \mathrm{~nm}$ and $576 \mathrm{~nm}$, respectively, their fluorescence spectra could be employed to monitor the compression-decompression behavior. A blue laser (405 nm) was used to excite the helicene molecules, and the actual hydrostatic pressure was calibrated by the fluorescence spectra of a small ruby ${ }^{[16]}$. As demonstrated in Figures $2 \mathrm{a}$ and $2 \mathrm{~d}$, the emission maxima of both molecules were gradually red-shifted when increasing the pressure up to $\sim 6 \mathrm{GPa}$, accompanied by a significant decrease in the fluorescence intensity. The fluorescence spectra resumed their original state by releasing the hydrostatic pressure to ambient conditions (Figures $2 b$ and $2 e$ ). High reversibility of the fluorescence response upon compression and decompression was observed in both [7] and [9] (Figure S3), indicating that the spectroscopic changes were caused by physical distortions of the molecular conformation and crystal packing rather than by chemical transformation or degradation. The pressure-dependent piezochromic process could also be visually observed with the fluorescence photographs captured by a digital camera. As shown in Figures $2 \mathrm{c}$ and $2 \mathrm{f}$, the crystals exhibited a gradual red-shift in color and a significant darkening of brightness upon compression. This was fully recovered by releasing the pressure, consistent with the spectroscopic observations.
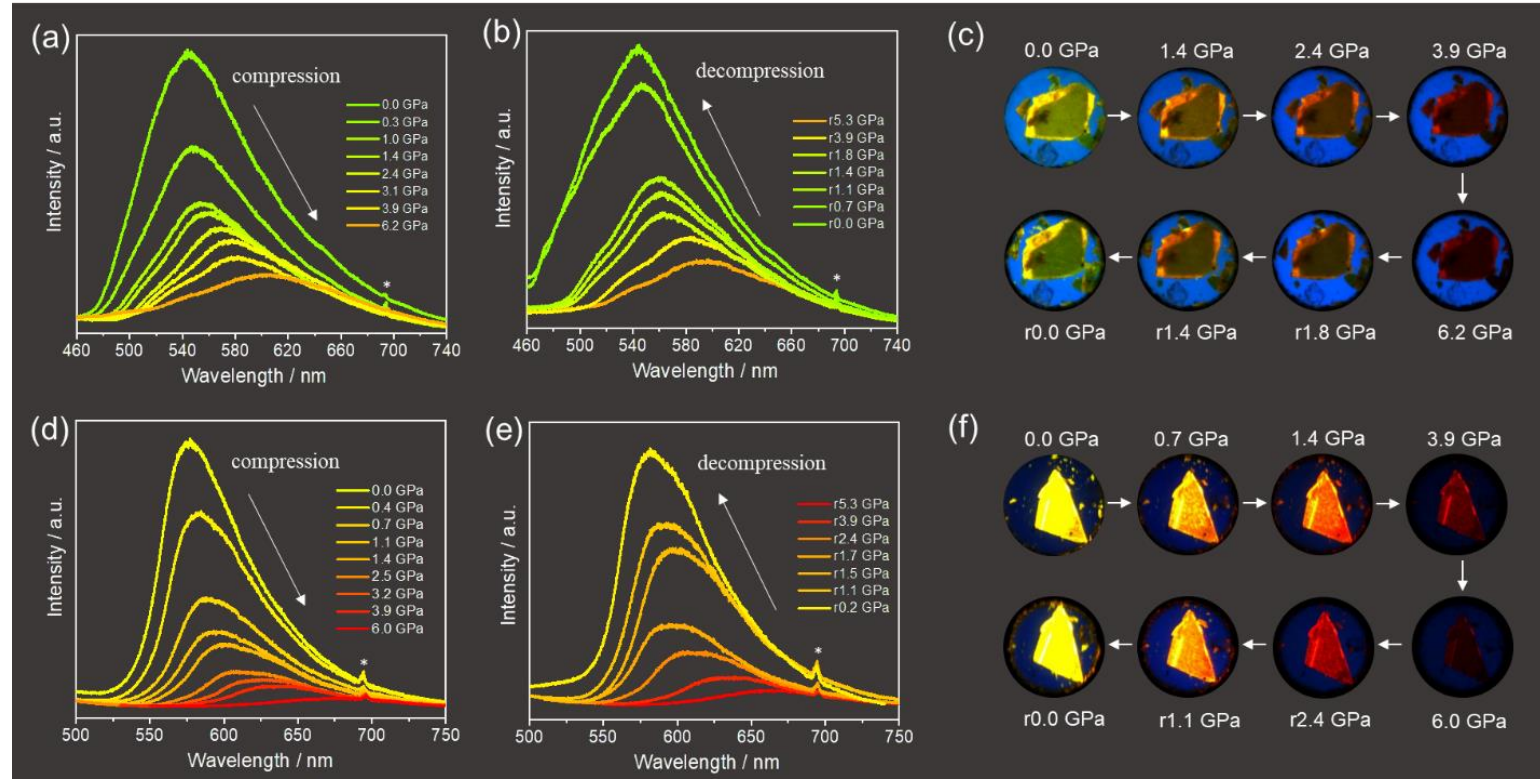

(f)

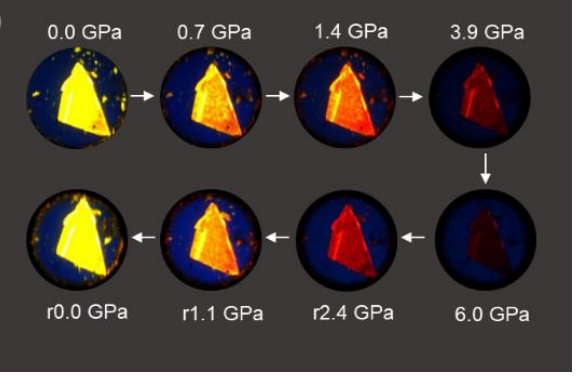

Figure 2. Fluorescence spectra change of [7] upon compression (a) and decompression (b), and the corresponding fluorescence images (c). Fluorescence spectra change of [9] upon compression (d) and decompression (e), and the corresponding fluorescence images (f). The ruby signal is marked with a star in the spectra.

More information about the pressure-induced changes of the [7] and [9] crystals was obtained by plotting the wavelength shift and emission intensity against the hydrostatic pressure in Figure 3. Interestingly, the shift of the wavelength exhibited an approximately linear relationship with the applied pressure for both molecules. The pressure-dependent wavelength shift in [9] was $~ 16.4 \mathrm{~nm} \mathrm{GPa}^{-1}$, which was larger than that of [7] with 10.5 nm GPa${ }^{-1}$. On the other hand, the fluorescence intensities decayed exponentially upon compression. The decrease in intensity could be attributed to stronger intermolecular interactions 
at a compressed distance under higher pressure, leading to suppression of the emission process ${ }^{[17]}$ or additional nonradiative decay pathways ${ }^{[6 b, 18]}$. Notably, [9] possessed a larger change in, both, wavelength shift and intensity decrease than [7]. In combination with the force constants discussed above (Figure S2), the fluorescence of the more rigid molecular spring [9] is more sensitive to external pressure. Compared with other organic piezochromic luminescent molecules in the literature[19], these $\pi$-extended helicenes exhibit large pressure-dependent wavelength shifts (Figure S4), which could be ascribed to the combination of inter- and intramolecular $\pi-\pi$ interactions (see below).
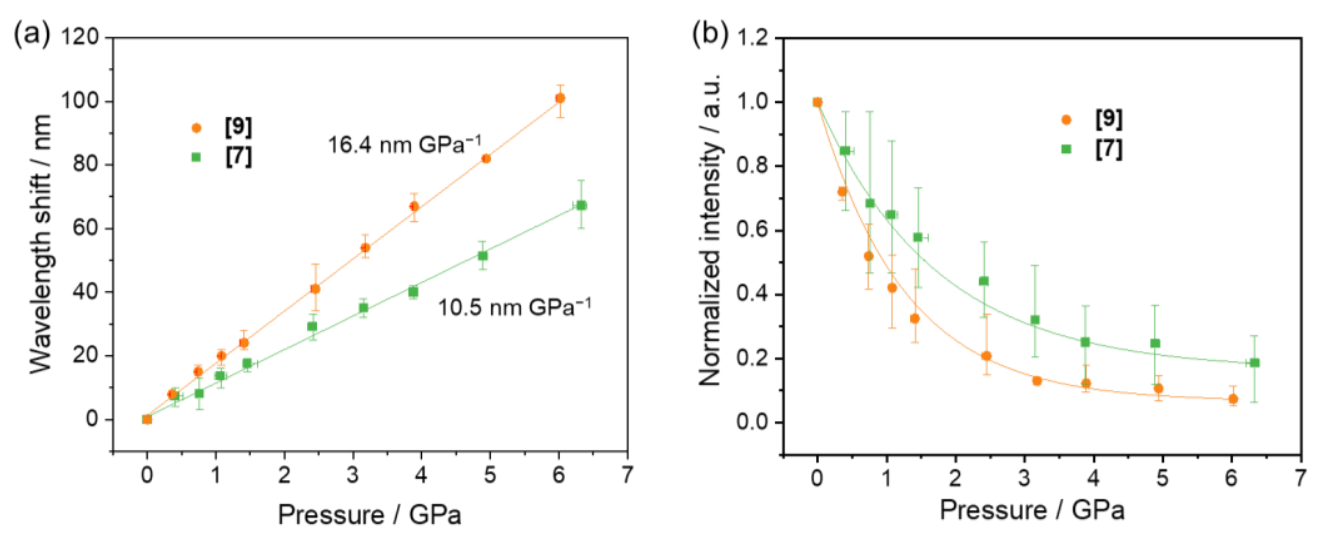

Figure 3. The fluorescence shift (a) and intensity change (b) of the [7] and [9] crystals under external pressure. The error bars are determined in 3-4 experiments. The intensities of the peaks are normalized by the initial data from starting pressure in the DAC.

It is widely accepted that the intermolecular packing in the crystal lattices becomes closer with increasing pressure ${ }^{[7]}$. Considering that the $\pi$-extended helicenes act as molecular springs, the extra intramolecular effect upon compression is also expected. However, it is challenging to quantitatively disentangle the inter- and intramolecular effects on the photophysical properties. To address the effect of intermolecular interactions on optical properties, the fluorescence spectra of their dilute solutions $\left(10^{-5} \mathrm{M}\right)$ and amorphous powders were investigated under ambient pressure (Figure S5). The amorphous powders were obtained by a freeze-drying process of the corresponding solutions, thus maintaining the molecular conformations as in dilute solutions. Compared to the crystalline state, the emission peaks of [7] and [9] in the amorphous state were hypsochromically shifted for both samples and were blue-shifted even further to 495 and $532 \mathrm{~nm}$ in solutions, respectively. The wavelength change is likely caused by the increased intermolecular distance and thus weakened $\pi-\pi$ interactions due to disaggregation ${ }^{[6 b, 13]}$. Therefore, on the opposite, tighter crystal stacking and stronger intermolecular $\pi-\pi$ interactions upon compression are considered to contribute to the red-shift of the emission in Figure 3a.

On the other hand, high-pressure experiments on dilute petroleum ether solutions of [7] and [9] were performed, where the photophysical properties of highly solvated molecules were measured to exclude the intermolecular effect. Similar to the crystals, the fluorescence maxima were gradually red-shifted and the intensity dramatically deceased upon compression (Figure S6 and S7). [9] again exhibited a larger band displacement than [7], although the changes occurred no longer in a linear way (Figure S8). Therefore, the decrease of intramolecular distance between the end of the helixes is believed to be another reason for the fluorescence red-shift under higher pressures. Notably, unlike the reversibility in the crystals (Figure 2), the fluorescence spectra in solutions did not return to their original state when releasing the pressure, probably because of stronger photobleaching ${ }^{[20]}$ in dilute solutions where molecules were fully exposed to laser irradiation. Unfortunately, the limited sample amount $(<1 \mu \mathrm{g})$ in our current DAC apparatus hindered further ex-situ analysis after high-pressure experiments. Nevertheless, the spectroscopic results in solution upon compression corroborate the general trend in crystals, suggesting contributions of intramolecular effects on the spectra red-shift in Figure 3a.

To better understand the behavior of $\pi$-extended helicenes upon compression, theoretical calculations were performed by DFT and QM/MM methods to simulate the cell parameters, electronic structures, and optical properties under pressure ${ }^{[21]}$. The cell parameters and atom positions of [7] and [9] crystals at ambient pressure ( $0 \mathrm{GPa}$ ) were obtained from their single-crystal data ${ }^{[15]}$, while those under different pressures were optimized by monotonically decreasing the cell volume with the extra pressure up to $8.0 \mathrm{GPa}$ based on Vienna ab initio simulation package ${ }^{[22]}$. Notably, the crystal lattices were calculated to continuously reduce with increasing pressure (Figure S11), which suggested that the crystal structures of [7] and [9] could be stably maintained. The possible phase transitions under compression, therefore, could be excluded according to the literature ${ }^{[23]}$. The absence of phase transition was also experimentally supported by the linear fluorescence shifts in Figure 3a. As demonstrated in Figures $4 \mathrm{a}$ and $4 \mathrm{~b}$, at ambient pressure in the crystalline state, the distance of helixes $r$, determined from the centroid-centroid distance of the overlapping benzene rings, is $3.95 \AA$ in [7], which is larger than that of [9] (3.54 $\AA$ ). Under 8.0 GPa, the $r$ of [7] and [9] was simulated to decrease by $0.49 \AA$ and $0.39 \AA$, respectively. This result indicates that [9] is a more rigid molecular spring under high pressure due to its larger $\pi-\pi$ overlap and stronger repulsion at the helix position, which is consistent with the initial force constants calculations in Figure S2. 
Multiscale crystal models were constructed to computationally investigate the optoelectronic properties of [7] and [9] in the excited states using the QM/MM method in the Gaussian package ${ }^{[21,24]}$ (Figure 4c). Based on the ground-state crystal structure and excited-state simulation, the experimental pressure-dependant emission of the [7] and [9] crystals could be reproduced. By applying the pressure up to $8 \mathrm{GPa}$, the calculated emission wavelength of [9] increased nearly linearly from $604 \mathrm{~nm}$ to $667 \mathrm{~nm}$ with a pressure-dependent wavelength shift of $\sim 8 \mathrm{~nm} \mathrm{GPa}{ }^{-1}$, being consistent with the trend observed experimentally (Figure 4e). Similarly, the simulated wavelength shift of [7] under different pressures in Figure 4d also agreed well with the experimental results with a smaller pressure-dependent wavelength shift $\left(\sim 3 \mathrm{~nm} \mathrm{GPa}^{-1}\right)$.

The changes of the fluorescence intensity can be modelled by the theoretical calculations of the radiative rate constant $k_{\mathrm{r}}$ using the Strickler-Berg equation ${ }^{[25]}$ :

$$
k_{r}=\frac{2 f \tilde{v}^{2}}{3}
$$

where $f$ is the oscillator strength and $\tilde{v}^{2}$ is the de-excitation energy from $\mathrm{S}_{1}$ to $\mathrm{S}_{0}$. The calculated $k_{\mathrm{r}}$ values of [7] and [9] under different pressures are summarized in Tables S1 and S2, suggesting that the radiative rate $k_{\mathrm{r}}$ of [9] is higher than that of [7], which explains the decreasing fluorescence intensity in Figure $3 b^{[15,25 b, 26]}$. More computational details are summarized in the supporting information (Figure S9-S13).

In summary, we reported the changes in the structure and luminescence of a pair of $\pi$-extended [ $n$ ]helicenes, [7] and [9], as molecular springs under high pressure. Based on the experimental results and theoretical calculations, [9] could be regarded as a more rigid molecular spring than [7] due to its larger $\pi-\pi$ overlap at the helical ends. In the high-pressure fluorescence measurements, both molecules exhibited a significant red-shift in emission wavelength and an exponential decay in fluorescence intensity, both in the crystalline and solution state. Although it is not possible to fully disentangle the inter- and intramolecular interactions, their influence on the emission was explored by investigating the spectra in dilute solutions and freeze-dried amorphous powders, proving a unique intramolecular $\pi-\pi$ effect in these spring-like molecules. The piezochromic behavior qualifies [9] for applications in more sensitive pressure sensing devices due to the large emission shift (>100 nm). Our results provide new insights into the structural effects of helicene molecules on their optical properties under high pressures and open up new opportunities to utilize them as molecular springs.

(a)
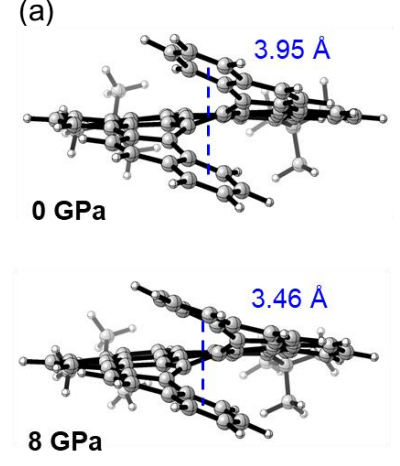

(d)

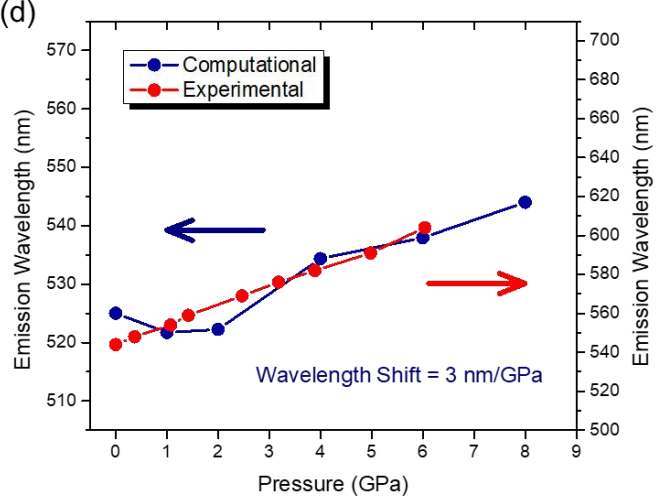

(b)

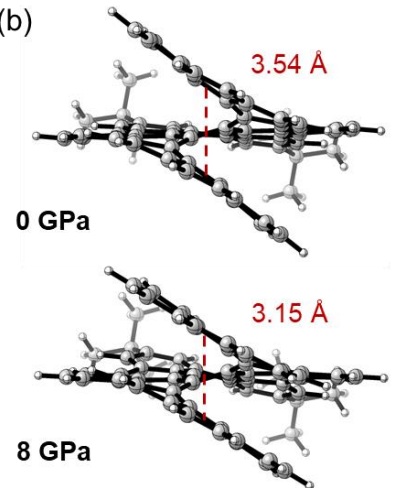

(c)

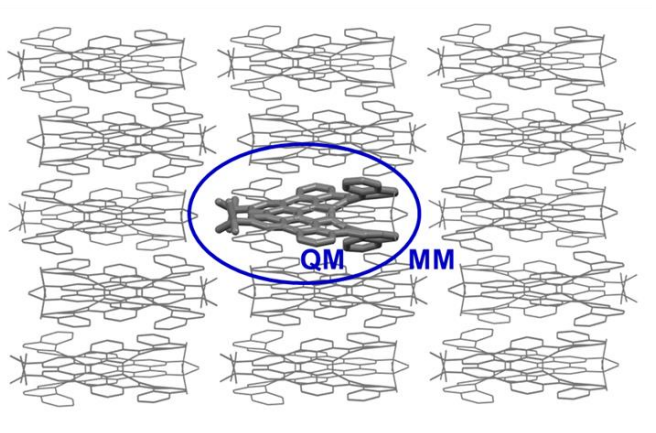

(e)

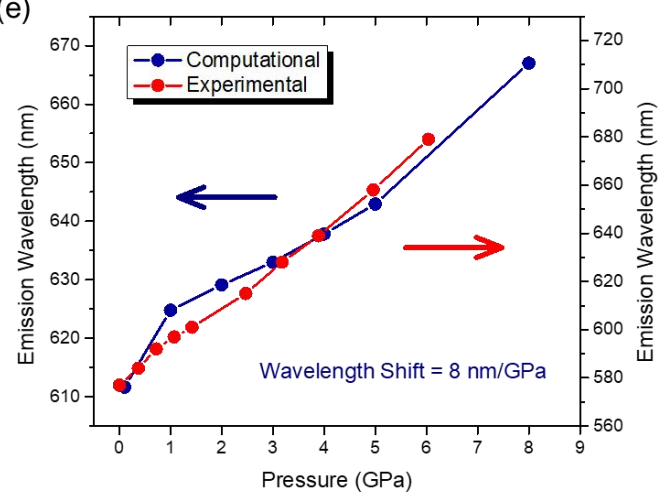

Figure 4. Molecular structure of (a) [7] and (b) [9] under ambient pressure and high pressure based on theoretical simulation. (c) Illustration of QM/MM model. The theoretical and experimental emission wavelength changes of (d) [7] and (e) [9] as a function of pressure. Excited-state structures were optimized at TD-CAM-B3LYP/6-31G(d):UFF level of theory, while the fluorescence emission was modelled with TD-PBE0/6-311G(d) level of theory.

\section{Acknowledgements}

This work was financially supported by the Max Planck Society. Z. Q. acknowledges financial support from the Alexander von Humboldt Foundation.

\section{Conflict of Interest}


The authors declare no conflict of interest.

Keywords: diamond anvil cell $\bullet$ high-pressure fluorescence $\bullet$ molecular spring $\bullet \pi$-extended helicene $\bullet$

[1] Y. Shen, C. F. Chen, Chem. Rev. 2012, 112, 1463- 1535.

[2] M. Gingras, Chem. Soc. Rev. 2013, 42, 1051 - 1095.

[3] C. B. Huang, A. Ciesielski, P. Samori, Angew. Chem. 2020, 132, 7387 - 7398; Angew. Chem. Int. Ed. 2020, 59, 7319 - 7330.

[4] a) Z. Zhou, X. Y. Wang, Z. Wei, K. Müllen, M. A. Petrukhina, Angew. Chem. 2019, 131, 15111- 15115; Angew. Chem. Int. Ed. 2019, 58, 14969 - 14973; b) Z. Zhou, L. Fu, Y. Hu, X. Y. Wang, Z. Wei, A. Narita, K. Müllen, M. A. Petrukhina, Angew. Chem. 2020, 132, 16057 - 16061; Angew. Chem. Int. Ed. 2020, 59, 15923 - 15927.

[5] Y. Nakakuki, T. Hirose, K. Matsuda, J. Am. Chem. Soc. 2018, 140, 15461 - 15469.

[6] a) D. Zhao, M. Wang, G. Xiao, B. Zou, J. Phys. Chem. Lett. 2020, 11, 7297- 7306. b) C. Wang, Z. Li, Mater. Chem. Front. $2017,1,2174-2194$.

[7] W. Cai, R. Zhang, Y. Yao, S. Deemyad, Phys. Chem. Chem. Phys. 2017, 19, 6216-6223.

[8] a) Y. Li, Z. Ma, A. Li, W. Xu, Y. Wang, H. Jiang, K. Wang, Y. Zhao, X. Jia, ACS Appl. Mater. Interfaces 2017, 9, 8910 - 8918; b) J. Wu, J. Tang, H. Wang, Q. Qi, X. Fang, Y. Liu, S. Xu, S. X. Zhang, H. Zhang, W. Xu, J. Phys. Chem. A 2015, 119, 9218 - 9224; C) Z. Zhao, X. Zheng, L. Du, Y. Xiong, W. He, X. Gao, C. Li, Y. Liu, B. Xu, J. Zhang, F. Song, Y. Yu, X. Zhao, Y. Cai, X. He, R. T. K. Kwok, J. W. Y. Lam, X. Huang, D. Lee Phillips, H. Wang, B. Z. Tang, Nat. Commun. 2019, 10, 2952.

[9] a) T. Ono, Y. Tsukiyama, A. Taema, H. Sato, H. Kiyooka, Y. Yamaguchi, A. Nagahashi, M. Nishiyama, Y. Akahama, Y. Ozawa, M. Abe, Y. Hisaeda, ChemPhotoChem 2018, 2, 416 - 420; b) A. Li, Z. Ma, J. Wu, P. Li, H. Wang, Y. Geng, S. Xu, B. Yang, H. Zhang, H. Cui, W. Xu, Adv. Opt. Mater. 2018, 6, 1700647.

[10] C. Liu, G. Xiao, M. Yang, B. Zou, Z. L. Zhang, D. W. Pang, Angew. Chem. 2018, 130, 1911-1915; Angew. Chem. Int. Ed. $2018,57,1893$ - 1897.

[11] T. Geng, C. Liu, G. Xiao, S. Lu, B. Zou, Mater. Chem. Front. 2019, 3, 2617 - 2626.

[12] a) P. F. Jones, M. Nicol, J. Chem. Phys. 1968, 48, 5440 - 5447; b) Y. Dai, H. Liu, T. Geng, F. Ke, S. Niu, K. Wang, Y. Qi, B. Zou, B. Yang, W. L. Mao, Y. Lin, J. Mater. Chem. C 2021, 9, $934-938$.

[13] K. Nagura, S. Saito, H. Yusa, H. Yamawaki, H. Fujihisa, H. Sato, Y. Shimoikeda, S. Yamaguchi, J. Am. Chem. Soc. 2013, $135,10322-10325$.

[14] S. Zhang, Y. Dai, S. Luo, Y. Gao, N. Gao, K. Wang, B. Zou, B. Yang, Y. Ma, Adv. Funct. Mater. 2017, 27,1602276.

[15] Z. Qiu, C. W. Ju, L. Frederic, Y. Hu, D. Schollmeyer, G. Pieters, K. Müllen, A. Narita, J. Am. Chem. Soc. 2021, 143, $4661-4667$.

[16] G. Shen, Y. Wang, A. Dewaele, C. Wu, D. E. Fratanduono, J. Eggert, S. Klotz, K. F. Dziubek, P. Loubeyre, O. V. Fat'yanov, P. D. Asimow, T. Mashimo, R. M. M. Wentzcovitch, High Pressure Res. 2020, 40, $299-314$.

[17] T. Hirose, N. Ito, H. Kubo, T. Sato, K. Matsuda, J. Mater. Chem. C 2016, 4, $2811-2819$.

[18] H. Yuan, K. Wang, K. Yang, B. Liu, B. Zou, J. Phys. Chem. Lett. 2014, 5, 2968 - 2973.

[19] a) Z. A. Dreger, H. Lucas, Y. M. Gupta, J. Phys. Chem. B 2003, 107, 9268 - 9274; b) K. Paudel, H. Knoll, M. Chandrasekhar, S. Guha, J. Phys. Chem. A 2010, 114, 4680 - 4688; c) X. Meng, G. Qi, X. Li, Z. Wang, K. Wang, B. Zou, Y. Ma, J. Mater. Chem. C, 2016, 4, 7584 - 7588; d) T. Geng, T. Feng, Z. Ma, Y. Cao, Y. Chen, S. Tao, G. Xiao, S. Lu, B. Yang, B. Zou, Nanoscale 2019, 11, $5072-5079 ;$ e) N. Li, Y. Gu, Y. Chen, L. Zhang, Q. Zeng, T. Geng, L. Wu, L. Jiang, G. Xiao, K. Wang, B. Zou, J. Phys. Chem. C 2019, 123, 6763 - 6767; f) R. Li, M. Wang, H. Zhao, Z. Bian, X. Wang, Y. Cheng, W. Huang, J. Phys. Chem. Lett. 2020, 11, $5896-5901$.

[20] a) A. P. Demchenko, Methods. Appl. Fluoresc. 2020, 8, 022001; b) T. Christ, F. Kulzer, P. Bordat, T. Basché, Angew. Chem. 2001, 113, 4323 4326; Angew. Chem. Int. Ed. 2001, 40, 4192 - 4195.

[21] a) H. Liu, Y. Gu, Y. Dai, K. Wang, S. Zhang, G. Chen, B. Zou, B. Yang, J. Am. Chem. Soc. 2020, 142, 1153 - 1158; b) X. Lu, Y. Sun, Z. Zhang, Z. Shuai, W. Hu, Chin. Chem. Lett. 2021, 32, 1233 - 1236; c) Q. Peng, H. Ma, Z. Shuai, Acc. Chem. Res. 2021, 54, 940 - 949.

[22] a) G. Kresse, D. Joubert, Phys. Rev. B 1999, 59, 1758 - 1775; b) G. Kresse, J. Furthmüller, Phys. Rev. B 1996, 54, $11169-11186$.

[23] T. Zhang, W. Shi, D. Wang, S. Zhuo, Q. Peng, Z. Shuai, J. Mater. Chem. C, 2019, 7, $1388-1398$.

[24] a) A. Warshel, M. Levitt, J. Mol. Biol. 1976, 103, 227 - 249; b) S. Difley, L.-P. Wang, S. Yeganeh, S. R. Yost, T. V. Voorhis, Acc. Chem. Res. 2010, 43, 995 - 1004; c) J. A. Elliott, Int. Mater. Rev. 2013, 56, 207 - 225.

[25] a) Z. Cao, Q. Zhang, J. Comput. Chem. 2005, 26, 1214 - 1221; b) A. W. Kohn, Z. Lin, T. Van Voorhis, J. Phys. Chem. C 2019, $123,15394-$ 15402.

[26] a) E. Jin, Q. Yang, C. W. Ju, Q. Chen, K. Landfester, M. Bonn, K. Müllen, X. Liu, A. Narita, J. Am. Chem. Soc. 2021, 143, 10403 - 10412; b) J. M. Fernandez-Garcia, P. J. Evans, S. Medina Rivero, I. Fernandez, D. Garcia-Fresnadillo, J. Perles, J. Casado, N. Martin, J. Am. Chem. Soc. 2018, 140, $17188-17196$.

\section{Entry for the Table of Contents}

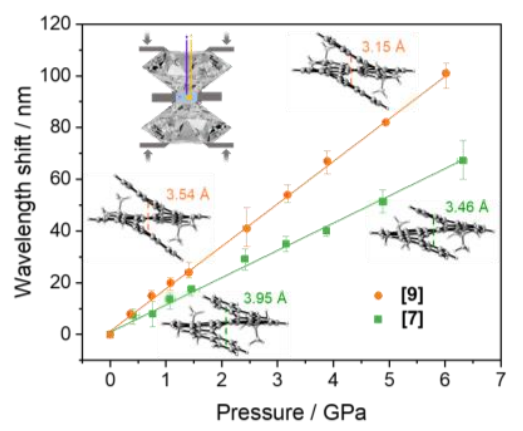


A pair of $\pi$-extended helicenes ( $n=7$ and 9) was selected as a model system for molecular springs to study the relationship between optical properties and molecular structures under high pressure in a diamond anvil cell. Based on experimental and simulation results, we found that the mechanical and fluorescent properties of these molecular springs are strongly influenced by the intramolecular $\pi-\pi$ interactions between their overlapping helixes. 